Towards a Theory of Search Queries

Non Peer-reviewed author version

FLETCHER, George H. L.; VAN DEN BUSSCHE, Jan; VAN GUCHT, Dirk \& VANSUMMEREN, Stijn (2009) Towards a Theory of Search Queries. In:

Proceedings of the Twelfth International Conference on Database Theory. p. 201-211..

Handle: http://hdl.handle.net/1942/9442 


\section{Towards a Theory of Search Queries}

\author{
George H.L. Fletcher \\ Washington State University \\ Vancouver \\ fletcher@vancouver.wsu.edu \\ Dirk Van Gucht \\ Indiana University \\ vgucht@cs.indiana.edu
}

\author{
Jan Van den Bussche \\ Hasselt University and \\ Transnational University of Limburg \\ jan.vandenbussche@uhasselt.be \\ Stijn Vansummeren* \\ Hasselt University and \\ Transnational University of Limburg \\ stijn.vansummeren@uhasselt.be
}

\begin{abstract}
The need to manage diverse information sources has triggered the rise of very loosely structured data models, known as "dataspace models." Such information management systems must allow querying in simple ways, mostly by a form of searching. Motivated by these developments, we propose a theory of search queries in a general model of dataspaces. In this model, a dataspace is a collection of data objects, where each data object is a collection of data items. Basic search queries are expressed using filters on data items, following the basic model of boolean search in information retrieval. We characterise semantically the class of queries that can be expressed by searching. We apply our theory to classical relational databases, where we connect search queries to the known class of fully generic queries, and to dataspaces where data items are formed by attribute-value pairs. We also extend our theory to a more powerful, associative form of searching where one can ask for objects that are similar to objects satisfying given search conditions. Such associative search queries are shown to correspond to a very limited kind of joins. Specifically, we show that the basic search language extended with associative search can define exactly the queries definable in a restricted fragment of the semijoin algebra working on an explicit relational representation of the dataspace.
\end{abstract}

\section{INTRODUCTION}

In most current information systems such as Web search engines, e-commerce sites, or desktop search systems, as well as in classical information retrieval systems such as library catalogs or document repositories, users query the database by means of a search interface. One can say that searching has become the norm. Searching is expressed by means of keywords which can be combined with boolean operators.

*Stijn Vansummeren is a Postdoctoral Fellow of the Research Foundation - Flanders (FWO).

Permission to copy without fee all or part of this material is granted provided that the copies are not made or distributed for direct commercial advantage, the ACM copyright notice and the title of the publication and its date appear, and notice is given that copying is by permission of the ACM. To copy otherwise, or to republish, to post on servers or to redistribute to lists, requires a fee and/or special permissions from the publisher, ACM. ICDT 2009, March 23-25, 2009, Saint Petersburg, Russia.

Copyright 2009 ACM 978-1-60558-423-2/09/0003 ...\$5.00
Such a basic search facility is much weaker than the standard database query languages, where select-project-join queries are considered the minimum. Indeed, fully-fledged firstorder logic is the norm (cf. Codd's relational algebra and calculus), and contemporary languages such as SQL/PSM or XQuery are even computationally complete.

Database queries are a major theme of database theory [3]. In general, queries are generic mappings from databases to relations, where 'generic' refers to invariance under isomorphisms $[4,10]$. Many classes of database queries have been identified and characterized in terms of semantic properties; expressibility in various query languages; or computability under various complexity limitations. Since searching is such a simple, natural, and important form of querying, it appears that search queries deserve to have their own chapter in the theory of database queries. Our goal in this paper is to propose a first draft of such a chapter. Apart from the foundational motivation, our work is further motivated by two important trends in data management research: dataspaces and usability.

Dataspaces $[11,12,17]$ are a new type of databases, characterized by a very loosely structured data model and geared towards the management of data coming from a diverse set of sources. Dataspaces are queried by a form of searching. In essence, the data in a dataspace is modeled as a collection of objects, where each object is a collection of attribute-value pairs. Dataspaces are queried by searching for conditions on attributes or values, or by following links between objects.

Improving the usability of database systems has been an issue pretty much since the beginning of database research, as witnessed, for example, by the past research on universal relation interfaces (surveyed by Ullman [24, Chapter 17]). Recently, Jagadish [18] has revived our interest in this topic; he argues, among other things, that queries involving explicit joins or subqueries are too cumbersome to express, and stimulates us to ask how far we can get with simpler forms of querying such as searching, or with more implicit or automatic ways of joining information.

So, in a nutshell, in this paper, we try to understand formally the question of how much database querying can be done using a basic search language, possibly extended with a simple facility for following links between objects.

We should also clarify what we do not do in this paper. We do not investigate how searching can be implemented efficiently. Rather, we focus on expressive power. Also, because of this focus, we ignore other important issues that have been investigated in research on keyword 
search in relational, tree-structured (XML), and semistructured (graph) databases [14, we give just one recent reference]. The two main such issues are automatically finding connections among objects in the database that contain the given keywords (which is a nice approach to the usability question mentioned above), and ranking the results of a keyword search.

Concretely, the contributions of this paper can be summarized as follows:

1. We define a general formal model of dataspaces, where a dataspace is a collection of data objects, and where an object is a collection of data items. On these data items, we assume a number of abstract filter predicates to be defined. These filters naturally serve as atomic search conditions: searching a dataspace with a filter returns all objects containing an item satisfying the filter. We obtain a basic search language by combining atomic searches using the boolean set operators union, intersection, and difference.

2. Search queries are defined in general as functions on dataspaces that map a dataspace to one of its subsets. The question then arises of exactly which such mappings are definable in the basic search language. This question turns out to be naturally answered by requiring the search queries to be invariant under a natural indistinguishability relation on objects. The concept of genericity (invariance) has always been a central theme in the development of the theory of database queries [3]; we have tried here to get at the right genericity concept for search queries.

3. We apply the above semantic characterization to the case of classical database relations. A relation can be viewed as a dataspace by viewing the tuples as sets of attribute-value pairs. When using as filters on such attribute-value pairs the classical selection conditions "attribute equals constant," we obtain as basic search queries precisely the class of search queries that are "fully generic" in the sense of Beeri, Milo and Ta-Shma $[6,7]$. (We hasten to add that these authors looked at full genericity of queries in a complex-object setting much richer than mere search queries on flat relations.)

4. We extend the basic model to allow for so-called "associative search", where one can search not just for all objects satisfying some search query, but also for all objects that are somehow linked to those objects. This amounts to adding a link operator to the basic search language, much in the spirit of modal logic $[8,9]$. Associative search is indeed a feature of most dataspace and keyword search query languages proposed in the literature $[11,12,14,17]$. Actually, in these proposals, links between objects are often assumed to be found automatically by the system. Since in this paper we are interested in a detailed analysis of expressive power, we focus on the case where the linking conditions are explicitly specified in the query.

5. A question we find interesting is how search query languages relate to standard database query languages. Those languages remain relevant in the search and dataspace setting. For example, SPARQL [2], the standard language to query RDF graphs [1], is closely re- lated to database queries on ternary relations $[13,16$, 22 ; RDF graphs can be viewed as a dataspace model.

Under the natural assumption that linking between two objects is done in terms of a set of similarity relations among the items in these objects, we can indeed relate associative search to standard database query languages. Specifically, we observe that associative search queries are definable in the semijoin algebra: the version of the relational algebra where the join operator is replaced by the semijoin $[20,21,23]$. Here, the semijoin algebra works on the natural representation of a dataspace as a binary relation; abstract filters are used as selection conditions, and abstract similarity predicates are used as join conditions.

Conversely, however, not every semijoin query is an associative search query. We actually identify three kinds of constructions that are definable in the semijoin algebra but not in our associative search language, and prove that the fragment of the semijoin algebra in which these three constructions are disallowed fully characterizes our associative search queries.

6. Finally, we show that our general theory is workable by applying it to the most common dataspace setting, where data items are attribute-value pairs. Unlike the application to classical relations mentioned earlier, here, there is no fixed schema and objects can have multiple values for the same attribute. We consider a natural repertoire of filters that can test if the attribute, and the value, equals, or is different from, a finite number of possibilities. In this setting, the semantic characterization of basic search queries can be rephrased in a very intuitive manner, as we will show. We will also instantiate the semijoin algebra characterization of associative search queries to the attribute-value setting. Associative search queries over attribute-value dataspaces are thus characterized as the queries expressible in a simple and attractive fragment of the semijoin algebra; this time, dataspaces are represented by ternary relations with schema (Oid, Att, Val), selection conditions are constant equalities (on attributes as well as on values; remember that there is no dataspace schema), and all semijoins are simple equijoins.

This paper is organized as follows. Section 2 presents the abstract model of search queries on dataspaces; Section 3 presents the application to classical database relations; Section 4 extends the abstract model with associative search; Section 5 gives the semijoin algebra characterization; and Section 6 presents the application to attribute-value dataspaces.

\section{BASIC MODEL}

We assume given a set $\mathcal{I}$ of abstract data elements called items.

Definition 1. An object over $\mathcal{I}$ is a finite, nonempty set of items. An abstract dataspace over $\mathcal{I}$ is a finite set of objects over $\mathcal{I}$.

For instance: 
- Items could be words over some alphabet. Objects then correspond to documents in the classical boolean model of information retrieval [5, chapter 4.2.3], and dataspaces correspond to collections of such documents. Figure 2(a) illustrates such a dataspace, consisting of two documents concerning events in St. Petersburg.

- Items could be pairs $(a, v)$ of attributes $a$ and values $v$. Objects, being finite sets of such pairs, then intuitively describe the attributes of a real-world entity. (Note that there can be multiple values for the same attribute.) A dataspace is just a collection of such descriptions. Figure 1, for example, depicts an attributevalue dataspace describing researchers, beers, and papers.

Under this concrete interpretation of $\mathcal{I}$, an abstract dataspace corresponds to the usual definition of a dataspace in the literature $[11,12,17]$. Indeed, while those definitions normally also include links (binary relationships) between objects, we can always represent such links using additional attributes. For example, to make a link authored by between a paper and an author, add an id attribute to the author, and add an attribute authored_by to the paper with the id of the author as value (multiple authors are no problem because objects in dataspaces can contain multiple attributevalue pairs for the same attribute).

- Staying with attribute-value pairs, we could consider those dataspaces whose objects contain only attributes from a fixed relation schema, and contain a single value for each such attribute. Such dataspaces are classical database relations.

We now define BSL: a basic boolean search language to query dataspaces. Formally, we assume a set $\mathcal{K}$ of predicate names called abstract keywords, and a $\mathcal{K}$-structure $(\mathcal{I}, \mathcal{M})$ on $\mathcal{I}$ in which every abstract keyword $k$ is interpreted as a unary predicate $\mathcal{M}(k)$ on $\mathcal{I}$, or, equivalently, a subset $\mathcal{M}(k) \subseteq \mathcal{I}$. The intuition for an item $i$ to be in $\mathcal{M}(k)$ is that $i$ "matches" $k$. For example, in the boolean information retrieval model, keywords could be predicates on words that test whether the word contains some fixed string as a substring. On attribute-value pairs, keywords could be predicates on pairs $(a, v)$ that test whether $a$ is some fixed attribute and $v$ is some fixed value.

Definition 2. The expressions of BSL are given by the grammar

$$
e \quad:=k \mid e \text { and } e \mid e \text { or } e \mid e \text { except } e,
$$

where $k$ ranges over the keywords in $\mathcal{K}$.

The semantics of these expressions is the following. An expression $e$ can be applied to a dataspace $D$, resulting in a subset $e(D)$ of $D$ defined as follows:

$$
\begin{aligned}
k(D) & :=\{o \in D \mid \exists i \in o: i \in \mathcal{M}(k)\} \\
\left(e_{1} \text { and } e_{2}\right)(D) & :=e_{1}(D) \cap e_{2}(D) \\
\left(e_{1} \text { or } e_{2}\right)(D) & :=e_{1}(D) \cup e_{2}(D) \\
\left(e_{1} \text { except } e_{2}\right)(D) & :=e_{1}(D)-e_{2}(D) .
\end{aligned}
$$

Note that the language is a bit redundant as $e_{1}$ and $e_{2}$ is equivalent to $e_{1}$ except $\left(e_{1}\right.$ except $\left.e_{2}\right)$.

It is important to appreciate the existential nature of the above-defined semantics. Thus, the expression $k_{1}$ except $k_{2}$ does not return all objects that contain an item that matches $k_{1}$ but not $k_{2}$; rather, it returns all objects that contain an item that matches $k_{1}$, but do not contain an item that matches $k_{2}$. Henceforth, for an object $o$ and a keyword $k$, we will write $o \mid=_{\mathcal{M}} k$ to denote that there exists $i \in o$ such that $i \in \mathcal{M}(k)$.

As just defined, every BSL expression defines a search query:

Definition 3. A search query is a mapping $q$ from dataspaces to dataspaces such that $q(D) \subseteq D$ for each $D$. A search query is definable in BSL if there exists an expression $e$ such that $e(D)=q(D)$ for every dataspace $D$.

Of course not all search queries are definable in BSL. Which ones are? We can answer this question by identifying three typical properties of BSL queries: additivity, $K$-safety, and $K$-distinguishing. We define these three properties next.

Definition 4. Let $q$ be a search query and let $K \subset \mathcal{K}$ be a finite set of keywords.

- We say that $q$ is additive if

$$
q(D)=\bigcup_{o \in D} q(\{o\})
$$

for any dataspace $D$.

- We say that $q$ is $K$-safe if for any dataspace $D$ and any $o \in q(D)$, we have that $o \models_{\mathcal{M}} k$ for at least one $k \in K$.

- Two objects $o_{1}$ and $o_{2}$ are called $K$-equivalent if for all $k \in K$ we have $o_{1} \models_{\mathcal{M}} k$ iff $o_{2} \models_{\mathcal{M}} k$. We denote this by $o_{1} \simeq_{K} o_{2}$. (Clearly, $\simeq_{K}$ is an equivalence relation.) We then say that $q$ is $K$-distinguishing if for any two dataspaces $D_{1}$ and $D_{2}$ and objects $o_{1} \in D_{1}$ and $o_{2} \in$ $D_{2}$ that are $K$-equivalent, we have $o_{1} \in q\left(D_{1}\right)$ iff $o_{2} \in$ $q\left(D_{2}\right)$.

The above three properties represent three distinctive features of BSL queries. Additivity simply states that the query can be processed one object at a time. $K$-safety states that we cannot retrieve arbitrary objects from the dataspace, but only objects that satisfy at least one of the specified keywords. This is also the case in all real-life search engines and information retrieval systems. Finally, $K$-distinguishing naturally states that the query can only distinguish between objects based on their satisfaction of specified keywords.

As a matter of fact, additivity already follows from $K$ distinguishing, since the latter property implies $o \in q(D)$ iff $o \in q(\{o\})$ which readily implies additivity. We stated the property of additivity separately because we will need it later independently of $K$-distinguishing.

We establish the following semantic characterization:

Theorem 5. A search query $q$ is definable in BSL if and only if $q$ is $K$-safe and $K$-distinguishing, for some finite set $K \subset \mathcal{K}$.

Proof. The only-if direction is straightforward; for $K$ we take the set of keywords occurring in the BSL expression. The properties of safety and distinguishing are then verified by structural induction (details omitted). For the ifdirection, we first observe that each $\simeq_{K}$ equivalence class $C$ is definable in BSL in the sense that we have an expression 

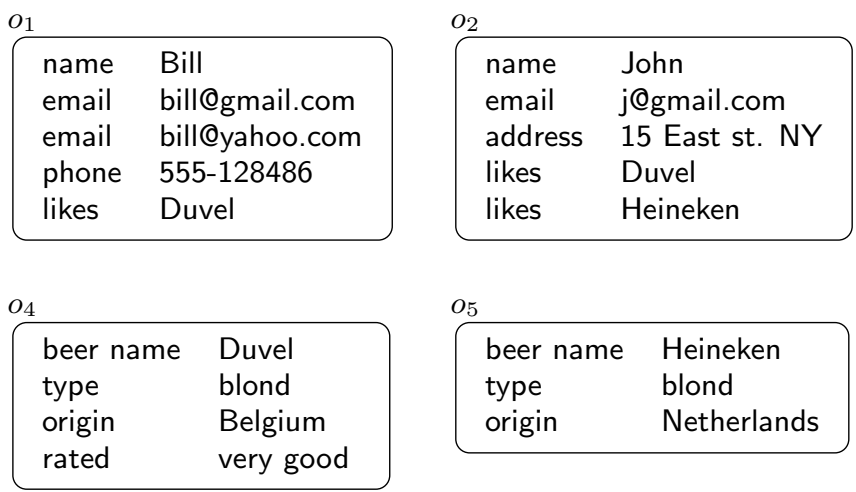

\begin{tabular}{|c|c|}
\hline $\begin{array}{l}\text { title } \\
\text { author } \\
\text { author } \\
\text { abstract } \\
\text { content } \\
\text { published in }\end{array}$ & $\begin{array}{l}\text { On the power ... } \\
\text { Bill } \\
\text { John } \\
\text { We investigate ... } \\
\ldots \\
\text { ICDT } 2009\end{array}$ \\
\hline \multicolumn{2}{|l|}{6} \\
\hline $\begin{array}{l}\text { conference } \\
\text { venue } \\
\text { year }\end{array}$ & $\begin{array}{l}\text { ICDT } 2009 \\
\text { St. Petersburg, RU } \\
2009\end{array}$ \\
\hline
\end{tabular}

Figure 1: An example attribute-value dataspace.

$e_{C}$ such that $o \in C$ iff $o \in e_{C}(\{o\})$. The only exception is the equivalence class of objects that do not satisfy any keyword in $K$; but since $q$ is $K$-safe that equivalence class can be ignored. Since $q$ is $K$-distinguishing, $q$ can then be defined as the union of all expressions $e_{C}$ over all $C$ for which $o \in q(\{o\})$ for $o \in C$. We omit the details.

We point out that in the presence of a wildcard keyword, the issue of $K$-safety becomes moot:

Corollary 6. If $\mathcal{K}$ includes a keyword $\star$ that matches any item (i.e., $\mathcal{M}(\star)=\mathcal{I}$ ), then a search query is definable in BSL iff it is $K$-distinguishing for some finite $K$.

We conclude this section by giving two illustrations of how one can show that certain queries are not definable in BSL.

Example 7. One may wonder if negations of keywords can be expressed: can we retrieve all objects containing an item that does not match some keyword $k$ ? The answer in general is no. In proof, suppose that $\mathcal{K}$ consists just of a single keyword $k$, with at least one matching item $i$ and at least one nonmatching item $j$. In addition, we can also use the wildcard. Then the query " $\neg k$ ", asking for all objects with an item that does not match $k$, is not $\{k\}$-distinguishing. ${ }^{1}$ Indeed, $\{i\} \simeq_{\{k\}}\{i, j\}$, but $\{i, j\}$ satisfies the query whereas $\{i\}$ does not. Hence, " $\neg k$ " is not definable in BSL.

Of course, one can add negated keywords directly to $\mathcal{K}$ and $\mathcal{M}$ to express such queries. One may then wonder whether that is enough to allow already all boolean combinations of keywords to be expressed. For example, can we now retrieve all objects containing an item that matches neither $k_{1}$ nor $k_{2}$ ? The answer is still no. In proof, suppose that $\mathcal{K}$ contains, besides the wildcard, two keywords $k_{1}$ and $k_{2}$ and their negations, with items $i_{1}, i_{2}, j_{0}$, and $j$, such that $i_{1}$ matches $k_{1}$ but not $k_{2} ; i_{2}$ matches $k_{2}$ but not $k_{1} ; j_{0}$ matches neither $k_{1}$ nor $k_{2}$; and $j$ matches both $k_{1}$ and $k_{2}$. Then the query " $\neg k_{1} \wedge \neg k_{2}$ " is not $K$-distinguishing with $K=\left\{k_{1}, k_{2}, \neg k_{1}, \neg k_{2}\right\}$ and thus not definable in BSL. Indeed, $\left\{i_{1}, i_{2}\right\} \simeq_{K}\left\{j_{0}, j\right\}$, but $\left\{j_{0}, j\right\}$ satisfies the query whereas $\left\{i_{1}, i_{2}\right\}$ does not.

\footnotetext{
${ }^{1}$ The attentive reader is assured that $\{k, \star\}$-distinguishing with $\star$ as in Corollary 6 is the same as $\{k\}$-distinguishing.
}

\section{APPLICATION TO CLASSICAL RELA- TIONS}

Note that the theory presented in Section 2 holds for any concrete interpretation of $\mathcal{I}, \mathcal{K}$, and $\mathcal{M}$. As an illustration of how this general theory can be applied, let us fix an infinite domain $\mathcal{V}$ of values and a relation schema $\Sigma$, i.e., a finite set of attributes. We can use the set of attribute-value pairs $\Sigma \times$ $\mathcal{V}$ as our set $\mathcal{I}$ of items. Then a tuple $t: \Sigma \rightarrow \mathcal{V}$ is an object over $\mathcal{I}$, and a finite relation $R$ over $\Sigma$ is a dataspace over $\mathcal{I}$. Note that conversely, not all objects over $\mathcal{I}$ are proper tuples, because in a proper tuple every attribute occurs, with only one value. Hence, not all dataspaces in this setting are proper relations. Let us denote the subclass of dataspaces over $\mathcal{I}$ that are finite relations over $\Sigma$ by $\mathcal{R}$.

As keywords we fix $\mathcal{K}=(\Sigma \times \mathcal{V}) \cup\{\star\}$, where each $(a, v) \in$ $(\Sigma \times \mathcal{V})$ is a literal keyword that matches only itself (i.e., $\mathcal{M}(a, v)=\{(a, v)\})$, and $\star$ is the wildcard keyword that matches all items (i.e., $\mathcal{M}(\star)=\mathcal{I}$ ). Note that such a literal keyword $(a, v)$ corresponds to the relational selection operator $\sigma_{a=v}$. Indeed, for all $D \in \mathcal{R}$ Definition 2 specializes to

$$
(a, v)(D)=\{t \in D \mid t(a)=v\}
$$

since $D$ consists only of tuples over $\Sigma$. So, in this setting, the language BSL evaluated over relations in $\mathcal{R}$ corresponds to the fragment of the relational algebra consisting of just the three operators of constant selection, union, and difference.

It now turns out that in this setting there is a connection between $K$-distinguishing search queries and fully $C$-generic queries. Fully generic queries without constants have been investigated (in a much richer setting than mere selection queries) by Beeri, Milo and Ta-Shma [6,7]; here we consider the version with constants. Thus, let $C \subset \mathcal{V}$ be a finite set of constants. A $C$-epimorphism is a mapping $f: \mathcal{V} \rightarrow \mathcal{V}$ such that both $\left.f\right|_{C}$ and $\left.f^{-1}\right|_{C}$ are the identity on $C$. We extend $f: \mathcal{V} \rightarrow \mathcal{V}$ to items, objects, and dataspaces in the canonical, pointwise manner:

$$
\begin{aligned}
f(a, v) & :=(a, f(v)), \\
f(o) & :=\{f(a, v) \mid(a, v) \in o\}, \\
f(D) & :=\{f(o) \mid o \in D\} .
\end{aligned}
$$

Now a search query $q$ is said to be fully $C$-generic (on the class $\mathcal{R}$ ) if for any relation $R$ over $\Sigma$ and any $C$-epimorphism $f$, we have $q(f(R))=f(q(R))$. We establish: 
Proposition 8. Let $C$ be a finite set of constants, and let $K=\{(a, v) \mid a \in \Sigma, v \in C\}$. Then a search query $q$ is $K$-distinguishing on the class $\mathcal{R}$ if and only if $q$ is additive and fully $C$-generic on $\mathcal{R}$.

Proof. The only-if direction is based on the fact that for any tuple $t$ and any $C$-epimorphism $f$, we have $t \simeq_{K} f(t)$. For the if-direction, assume $t \simeq_{K} t^{\prime}$. We can find a tuple $u$ and $C$-epimorphisms $f$ and $f^{\prime}$ such that $f(u)=t$ and $f^{\prime}(u)=t^{\prime}$. We then have

$$
\begin{aligned}
t \in q(R) & \Leftrightarrow t \in q(\{t\}) \\
& \Leftrightarrow f(u) \in q(\{f(u)\}) \\
& \Leftrightarrow u \in q(\{u\}) \\
& \Leftrightarrow f^{\prime}(u) \in q\left(\left\{f^{\prime}(u)\right\}\right) \\
& \Leftrightarrow t^{\prime} \in q\left(\left\{t^{\prime}\right\}\right) \\
& \Leftrightarrow t^{\prime} \in q\left(R^{\prime}\right) .
\end{aligned}
$$

From the above proposition and Corollary 6 we then obtain:

Corollary 9. A search query $q$ on relations over $\Sigma$ is definable in the relational algebra using only the operators constant selection, union, and difference, if and only if $q$ is additive and fully $C$-generic for some finite set $C$ of constants.

The main purpose of this modest theorem is to illustrate that our general theory can be effectively applied and connected to earlier work.

\section{ASSOCIATIVE SEARCH}

Since BSL queries are additive, BSL cannot define queries that relate objects to other objects; BSL cannot do joins. For example, in the boolean information retrieval setting (where $\mathcal{I}$ is a set of words and objects correspond to documents), the search query

"retrieve all documents that share a word with a document in which ICDT 2009 occurs"

is not additive, and therefore not definable in BSL. In dataspace systems, however, we want to be able to retrieve not just all objects that satisfy some query, but also all objects that are related to those objects $[11,12]$.

To this end, we extend our theory by further assuming a set $\mathcal{L}$ of abstract link conditions and extending our structure $\mathcal{M}$ to interpret these conditions as binary relationships between objects. So, for each $\lambda \in \mathcal{L}$, we have $\mathcal{M}(\lambda) \subseteq \mathcal{O} \times \mathcal{O}$, where $\mathcal{O}$ is the set of all objects.

We now define our associative search language ASL as an extension of the basic search language BSL with an operator for retrieving related objects:

Definition 10. The expressions of ASL are given by the grammar

$$
e \quad:=k \mid e \text { and } e \mid e \text { or } e \mid e \text { except } e \mid \operatorname{link}\langle\lambda\rangle e,
$$

where $\lambda$ ranges over $\mathcal{L}$. The semantics of the new construct is given by

$$
(\operatorname{link}\langle\lambda\rangle e)(D):=\left\{o \in D \mid \exists o^{\prime} \in e(D):\left(o, o^{\prime}\right) \in \mathcal{M}(\lambda)\right\} .
$$

Example 11. For example, in the boolean information retrieval setting, we might have a link condition relevant, interpreted as the relationship "document $o_{1}$ is relevant to document $\mathrm{O}_{2}$ " (computed according to some IR algorithm). Then the expression

$$
\text { link〈relevant }\rangle \text { ('ICDT' or 'St.Petersburg') }
$$

retrieves all documents that are relevant to documents containing the keywords 'ICDT' or 'St.Petersburg'.

There is an obvious connection between ASL and modal logic; indeed, ASL is a modal logic. We can make this connection precise by defining a bisimulation notion appropriate for ASL.

Definition 12. A pointed dataspace is a pair $(D, o)$ with $D$ a dataspace and $o$ an object in $D$. Let $K \subseteq \mathcal{K}$ and $L \subseteq \mathcal{L}$ be sets of keywords and link conditions, respectively. Two pointed dataspaces $(D, o)$ and $\left(D^{\prime}, o^{\prime}\right)$ are $n$ bisimilar, or $n$-bisimulation equivalent, under $K$ and $L$, denoted $(D, o) \rightleftarrows_{n}^{K, L}\left(D^{\prime}, o^{\prime}\right)$, if $o \simeq_{K} o^{\prime}$ and the following conditions hold for $n>0$ :

Forth: For any $\lambda \in L$, if $(o, p) \in \mathcal{M}(\lambda)$ for some $p \in D$, then there is some $p^{\prime} \in D^{\prime}$ such that $\left(o^{\prime}, p^{\prime}\right) \in \mathcal{M}(\lambda)$ and $(D, p) \rightleftarrows{ }_{n-1}^{K, L}\left(D^{\prime}, p^{\prime}\right)$.

Back: For any $\lambda \in L$, if $\left(o^{\prime}, p^{\prime}\right) \in \mathcal{M}(\lambda)$ for some $p^{\prime} \in D^{\prime}$, then there is some $p \in D$ such that $(o, p) \in \mathcal{M}(\lambda)$ and $(D, p) \rightleftarrows{ }_{n-1}^{K, L}\left(D^{\prime}, p^{\prime}\right)$.

We then obtain the following lemma known from the model theory of modal logic [15, Theorem 32]:

Lemma 13. Let $K$ be a finite nonempty set of keywords and let $L$ be a finite nonempty set of link conditions. Let $\operatorname{ASL}[K, L]$ denote the set of ASL expressions using keywords in $K$ and link conditions in $L$. Then the following are equivalent for any search query $q$ and any class $\mathcal{D}$ of dataspaces:

1. $q$ is definable on $\mathcal{D}$ in $\operatorname{ASL}[K, L]$ by an expression $e$ with nesting depth of link operators at most $n$. (That is, $e(D)=q(D)$ for all $D \in \mathcal{D}$.)

2. $q$ is ${ }_{n}^{K, L}$-invariant on $\mathcal{D}$, i.e., if $(D, o) \rightleftarrows{ }_{n}^{K, L}\left(D^{\prime}, o^{\prime}\right)$ with $D$ and $D^{\prime}$ in $\mathcal{D}$, then $o \in q(D)$ iff $o^{\prime} \in q\left(D^{\prime}\right)$.

We will put this lemma to good use later; now, we discuss the more pressing issue of how link conditions should actually be defined. After all, a flexible search query language should allow the link conditions to be expressed within the language itself. In section 6 we will look at how this can be done for the concrete case of attribute-value dataspaces, but here we will look at an intermediate level where link conditions are expressed on the level of, still abstract, relationships between items rather than objects.

So, assume now a set $\mathcal{S}$ of similarity relation names, or simrels for short. Each simrel $\sim$ is interpreted as a binary relation $\mathcal{M}(\sim) \subseteq \mathcal{I} \times \mathcal{I}$ on items. We now define the following basic class of link conditions, based on simrels and keyword search:

Definition 14. Let $k$ and $l$ be keywords and let $\sim$ be a simrel. Then the expression $k \sim l$ is called a simlink and can be used as a link condition with the following semantics:

$$
\begin{aligned}
\mathcal{M}(k \sim l)=\left\{\left(o, o^{\prime}\right)\right. & \in \mathcal{O} \times \mathcal{O} \mid \exists i \in o: \exists j \in o^{\prime}: \\
& i \in \mathcal{M}(k) \text { and } j \in \mathcal{M}(l) \text { and } i \sim j\}
\end{aligned}
$$




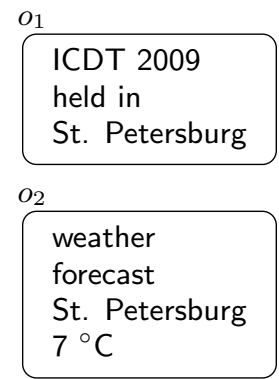

(a) Dataspace $D$

\begin{tabular}{|ll|}
\hline id & item \\
\hline \hline$o_{1}$ & ICDT 2009 \\
$o_{1}$ & held in \\
$o_{1}$ & St. Petersburg \\
$o_{2}$ & weather \\
$o_{2}$ & forecast \\
$o_{2}$ & St. Petersburg \\
$o_{2}$ & $7^{\circ} \mathrm{C}$ \\
\hline
\end{tabular}

(b) Binary relation $\operatorname{rep}(D)$
Figure 2: A dataspace and its relational representation.

The intuition behind simlinks is very natural. We search object $o$ on keyword $k$; we search object $o^{\prime}$ on keyword $l$; we compare the two search results and require that they contain a pair of similar items.

Example 15. For a simple example, in the boolean information retrieval setting, we might have a simrel soviet between words such that $w_{1}$ soviet $w_{2}$ if $w_{1}$ is a location name (city name, street name) from the Soviet era, and $w_{2}$ is the corresponding post-Soviet name. For example, Leningrad soviet St. Petersburg. Then the expression

$$
\text { link }\langle\star \text { soviet } \star\rangle \text { ('ICDT') }
$$

retrieves all documents containing Soviet versions of locations names mentioned in documents about ICDT. In Section 6 we will see more examples of simlinks.

For the remainder of this paper, we will always use the language ASL with simlinks as link conditions.

\section{SEMIJOIN ALGEBRA}

Acknowledging that search queries are a special kind of database queries, it is natural to ask how the language ASL (with simlinks) compares to more standard query languages. Observing that the link operator is a kind of semijoin, a comparison with the semijoin algebra seems a good approach to this question. The semijoin algebra is the version of the relation algebra where the join operator is replaced by the semijoin operator $[20,21]$.

Since the relational algebra works on relations, we need a relational representation of a dataspace:

Definition 16. For each dataspace $D$ let $\operatorname{rep}(D)$ be the binary relation $\{(o, i) \mid o \in D$ and $i \in o\}$ over the relation schema $\{$ id, item $\}$.

The objects in the id-column of this relation are regarded as object identifiers; the relational algebra is not able to peek inside the objects in any other way than working with the item column. An example is shown in Figure 2.

In the version of the semijoin algebra we are using, keywords on items are used as selection conditions, and simrels on items and equality on objects (viewed as id's) are used as semijoin conditions. The relation symbol $T$ (for 'Table') stands for the binary representation of the input dataspace. Every expression has an output schema that is either empty, the schema $\{$ id, item $\}$ itself, or one of the unary schemas $\{$ id $\}$ or $\{$ item $\}$ (we do not have renaming). Expressions must be

$$
\begin{aligned}
& \frac{E: \Sigma \quad \text { item } \in \Sigma \quad k \in \mathcal{K}}{\sigma_{k}(E): \Sigma} \\
& \frac{E: \Sigma \Delta \subseteq \Sigma}{\pi_{\Delta}(E): \Delta} \quad \frac{E_{1}: \Sigma E_{2}: \Sigma}{E_{1} \cup E_{2}: \Sigma} \quad \frac{E_{1}: \Sigma E_{2}: \Sigma}{E_{1}-E_{2}: \Sigma} \\
& \frac{E_{1}: \Sigma_{1} \quad E_{2}: \Sigma_{2} \quad \Sigma_{1} \cap \Sigma_{2}=\{\mathrm{id}\}}{E_{1} \ltimes E_{2}: \Sigma_{1}} \\
& \frac{E_{1}: \Sigma_{1} \quad E_{2}: \Sigma_{2} \quad \Sigma_{1} \cap \Sigma_{2}=\{\text { item }\} \quad \sim \in \mathcal{S}}{E_{1} \underset{\sim}{\ltimes} E_{2}: \Sigma_{1}} \\
& \frac{E_{1}:\{\text { id, item }\} \quad E_{2}:\{\text { id, item }\} \sim \in \mathcal{S}}{E_{1} \ltimes E_{2}:\{\text { id, item }\}}
\end{aligned}
$$

Figure 3: Syntax of SA.

well-typed, e.g., we only allow the union of two relations over the same schema. The full syntax of semijoin algebra (abbreviated SA) expressions, together with the derivation of their output schemas, is now given in Figure 3. The notation $E: \Sigma$ denotes that $E$ is a legal expression with output schema $\Sigma$.

The semantics of projection $\pi$, union $\cup$ and difference is well known; the semantics of the semijoin operators is as follows:

$$
\begin{aligned}
\sigma_{k}(R):= & \{t \in R \mid t(\text { item }) \in \mathcal{M}(k)\} \\
R_{1} \ltimes R_{2}:= & \left\{t_{1} \in R_{1} \mid \exists t_{2} \in R_{2}: t_{2}(\text { id })=t_{1}(\text { id })\right\} \\
R_{1} \ltimes R_{2}:= & \left\{t_{1} \in R_{1} \mid \exists t_{2} \in R_{2}:\right. \\
& \left.\left(t_{1}(\text { item }), t_{2}(\text { item })\right) \in \mathcal{M}(\sim)\right\} \\
R_{1} \ltimes R_{2}:= & \left\{(o, i) \in R_{1} \mid \exists j:\right. \\
=, \sim & \left.(o, j) \in R_{2} \text { and }(i, j) \in \mathcal{M}(\sim)\right\}
\end{aligned}
$$

So, $\ltimes$ is a normal natural semijoin on the common id attribute; $\ltimes \sim$ is a $\sim$-semijoin on the common item attribute; and $\ltimes=, \sim$ is a combination of the two.

Definition 17. A search query $q$ is definable in SA if there exists an SA expression $E: \Sigma$ with id $\in \Sigma$ such that $q(D)=$ $\pi_{\text {id }}(E)(\operatorname{rep}(D))$, for all dataspaces $D$. We also say that $E$ defines $q$ in this case.

The following is now expected:

Proposition 18. Each search query defineable in ASL(with simlinks) is definable in $\mathrm{SA}$.

Proof. Here is a straight syntactic translation:

$$
\begin{aligned}
\mathrm{SA}[k] & :=\sigma_{k}(T) \\
\mathrm{SA}\left[e_{1} \text { or } e_{2}\right] & :=\pi_{\text {id }} \mathrm{SA}\left[e_{1}\right] \cup \pi_{\text {id }} \mathrm{SA}\left[e_{2}\right] \\
\mathrm{SA}\left[e_{1} \text { except } e_{2}\right]: & =\pi_{\text {id }} \mathrm{SA}\left[e_{1}\right]-\pi_{\text {id }} \mathrm{SA}\left[e_{2}\right] \\
\mathrm{SA}[\operatorname{link}\langle k \sim l\rangle e] & :=\pi_{\text {id }}\left(\mathrm{SA}[k] \ltimes \sim \pi_{\text {item }}\left(\mathrm{SA}[l] \ltimes \pi_{\text {id }} \mathrm{SA}[e]\right)\right)
\end{aligned}
$$

Is the converse true as well? The answer is no, and we will perform a thorough analysis of the situation, with the goal of 
arriving at a well-defined fragment of SA that is equivalent to ASL.

The first observation is that SA can express boolean combinations of keywords. For example, the SA expression $\pi_{\text {id }}\left(T-\sigma_{k}(T)\right)$ defines the negated keyword $\neg k$, i.e., the query $q(D)=\{o \in D \mid \exists i \in o: i \notin \mathcal{M}(k)\}$. We have already seen in Example 7 that negated keywords, and more generally, boolean combinations of keywords, are not definable in ASL. This is easily repaired by closing the keywords under the boolean operators. Syntactically, we move from $\mathcal{K}$ to $\mathcal{K}^{*}$ which is the smallest set of keywords containing $\mathcal{K}$ and closed under the syntactic operators $\neg$ and $\vee$; semantically, we extend the interpretation $\mathcal{M}$ of $\mathcal{K}$ to an interpretation $\mathcal{M}^{*}$ of $\mathcal{K}^{*}$ in the natural way. Note that $\mathcal{K}^{*}$ necessarily includes a wildcard keyword $\star$ that matches all items. We can then revise Proposition 18 as follows:

Proposition 18 revised. Every search query defineable in ASL over $\mathcal{M}^{*}$ can be defined in $S A$ over $\mathcal{M}$.

Proof. The syntactic translation from the proof of Proposition 18 can be extended as follows: ( $\varphi$ and $\psi$ stand for boolean combinations of keywords)

$$
\begin{aligned}
\mathrm{SA}[k] & :=\sigma_{k}(T) \\
\mathrm{SA}[\neg \varphi] & :=T-\mathrm{SA}[\varphi] \\
\mathrm{SA}[\varphi \vee \psi] & :=\operatorname{SA}[\varphi] \cup \mathrm{SA}[\psi]
\end{aligned}
$$

Note that a boolean closed set of keywords automatically includes a wildcard keyword, in the form of $k \vee \neg k$.

The next proposition points at a number of distinct query constructions definable in SA but not in ASL:

Proposition 19. Each of the following $S A$ queries is not definable in $A S L$, even over the boolean closure of keywords:

$$
\begin{aligned}
& E_{1}:=\pi_{\text {id }}\left(\sigma_{k}(T) \underset{=, \sim}{\ltimes} \sigma_{l}(T)\right) \\
& E_{2}:=\pi_{\text {id }}\left(T-T \underset{\sim}{\ltimes} \pi_{\text {item }} \sigma_{k}(T)\right) \\
& E_{3}:=\pi_{\text {id }}\left(\sigma_{k}(T) \underset{\sim}{\ltimes} \pi_{\text {item }}\left(\sigma_{l}(T) \underset{\sim}{\ltimes} \pi_{\text {item }} \sigma_{m}(T)\right)\right) \\
& E_{4}:=\pi_{\text {id }}\left(T \underset{\sim}{\ltimes}\left(\pi_{\text {item }}(T)-\pi_{\text {item }}\left(T \ltimes \pi_{\text {id }} \sigma_{k}(T)\right)\right)\right)
\end{aligned}
$$

More precisely, for each of these queries there exists a simple interpretation $\mathcal{M}$ of the keywords $k, l$ and $m$ and the simrel $\sim$ such that the query is not definable in ASL over $\mathcal{M}^{*}$.

Note that, referring to the items $i \in \mathcal{M}(k)$ as $k$-items, the above four expressions define the following queries:

1. Retrieve all objects containing a $k$-item $i$ and an $l$-item $j$ such that $i \sim j$.

2. Retrieve all objects containing an item that is not $\sim$ to any $k$-item in the dataspace.

3. Retrieve all objects containing a $k$-item that is $\sim$ to some $l$-item $j$ in the dataspace, and $j$ itself is $\sim$ to some $m$-item in the dataspace.

4. Retrieve all objects containing an item $i$ that is $\sim$ to an item that is not present in any object containing a $k$-item.

These queries are shown to be undefinable in ASL by showing that they are not bisimulation invariant, then invoking Lemma 13.
Proof of Proposition 19. For the query $q_{1}$ expressed by $E_{1}$, we put $\mathcal{M}(k)=\left\{a_{1}, a_{2}\right\}$ and $\mathcal{M}(l)=\left\{b_{1}, b_{2}\right\}$, with $a_{1} \sim b_{1}$ and $a_{2} \sim b_{2}$. Now consider $D=\{o\}$ and $D^{\prime}=\left\{o_{1}, o_{2}\right\}$ with $o=\left\{a_{1}, b_{1}\right\} ; o_{1}=\left\{a_{1}, b_{2}\right\} ;$ and $o_{2}=\left\{a_{2}, b_{1}\right\}$. Then $(D, o)$ and $\left(D^{\prime}, o_{1}\right)$ are bisimilar $(n$-bisimilar for any $n)$. Yet, $o \in q_{1}(D)$ whereas $o_{1} \notin q_{1}\left(D^{\prime}\right)$.

For the query $q_{2}$ expressed by $E_{2}$, we use items $a, a^{\prime}$, and $b$ with $\mathcal{M}(k)=\{b\}$ and $a^{\prime} \sim b$. Now consider $D=\left\{o_{1}, o_{2}\right\}$ and $D^{\prime}=\left\{o_{1}^{\prime}, o_{2}\right\}$ with $o_{1}=\left\{a, a^{\prime}\right\} ; o_{2}=\{b\} ;$ and $o_{1}^{\prime}=$ $\left\{a^{\prime}\right\}$. Then $\left(D, o_{1}\right)$ is bisimilar to $\left(D^{\prime}, o_{1}^{\prime}\right)$, but $o_{1} \in q_{2}(D)$ whereas $o_{1}^{\prime} \notin q_{2}\left(D^{\prime}\right)$.

For the query $q_{3}$ expressed by $E_{3}$, we put $\mathcal{M}(k)=\{a\}$; $\mathcal{M}(l)=\left\{b, b_{1}, b_{2}\right\} ;$ and $\mathcal{M}(m)=\{c\}$, with $a \sim b, b \sim c$, $a \sim b_{1}$, and $b_{2} \sim c$. Now consider $D=\left\{o_{1}, o_{2}\right\}$ and $D^{\prime}=$ $\left\{o_{1}, o_{2}\right\}$ with $o_{1}=\{a\} ; o_{2}=\{b, c\} ;$ and $o_{2}^{\prime}=\left\{b_{1}, b_{2}, c\right\}$. Then $\left(D, o_{1}\right)$ is bisimilar to $\left(D^{\prime}, o_{1}\right)$, but $o_{1} \in q_{3}(D)$ whereas $o_{1} \notin q_{3}\left(D^{\prime}\right)$.

For the query $q_{4}$ expressed by $E_{4}$, we use items $a, b$ and $c$, with $\mathcal{M}(k)=\{a\}$, and $\sim$ is interpreted as equality. Now consider $D=\left\{o_{1}, o_{2}\right\}$ and $D^{\prime}=\left\{o_{1}^{\prime}, o_{2}\right\}$ with $o_{1}=\{b, c\}$; $o_{2}=\{a, b\} ;$ and $o_{1}^{\prime}=\{b\}$. Then $\left(D, o_{1}\right)$ is bisimilar to $\left(D^{\prime}, o_{1}^{\prime}\right)$, but $o_{1} \in q_{4}(D)$ whereas $o_{1}^{\prime} \notin q_{4}\left(D^{\prime}\right)$.

The above proposition can inspire us to restrict SA so as to obtain a fragment equivalent to ASL. $E_{1}$ suggests that we should banish the combined semijoin $\ltimes=, \sim \cdot E_{2}$ suggests that we should not allow unrestricted use of the result of a $\ltimes_{\sim}$ semijoin; we should project the result. But $E_{3}$ shows we should not project on $\{$ item $\}$, so we conclude we must always project the result of $\ltimes \sim$ on $\{$ id $\}$. Moreover, $E_{4}$ suggests that we should not allow projection on $\{$ item $\}$ altogether, except of course to allow for a semijoin $\ltimes_{\sim}$ to be applied. We thus arrive at the following fragment of SA, which we denote by $\mathrm{SA}^{\text {search }}$.

Definition 20. The fragment $\mathrm{SA}^{\text {search }}$ of $\mathrm{SA}$ is defined by the following rules:

- The operators $\ltimes_{=, \sim}, \pi_{\{\text {item }\}}$ and $\pi_{\emptyset}$ are disallowed.

- The rule for $\ltimes_{\sim}$ is changed as follows:

$$
\frac{E_{1}:\{\text { id }, \text { item }\} \quad E_{2}:\{\text { id }, \text { item }\} \quad \sim \in \mathcal{S}}{\pi_{\{\text {id }\}}\left(E_{1} \underset{\sim}{\ltimes} \pi_{\{\text {item }\}} E_{2}\right):\{\text { id }\}}
$$

We now establish the connection between associative search and the semijoin algebra:

Theorem 21. A search query is definable in $\mathrm{SA}^{\text {search }}$ over $\mathcal{M}$ if and only if it is definable in $A S L$ (with simlinks) over $\mathcal{M}^{*}$.

Proof. The if-direction follows from the observation that the translation from ASL to SA given in the proof of Proposition 18 stays within the fragment $\mathrm{SA}^{\text {search }}$.

The only-if direction is also proven by a translation, but a complication here is the translation of subexpressions with output schema $\{$ id, item $\}$, as such intermediate results are not directly representable by the result of a search query (which can only return id's).

Formally, for each $\mathrm{SA}^{\text {search }}$ expression $E$ we construct a finite set $\chi_{E}$ of pairs $(e, k)$, with $e$ an ASL expression and $k \in K^{*}$, such that for all $D$ : 
- if the output schema of $E$ is $\{$ id, item $\}$, then $E(\operatorname{rep}(D))$ equals

$$
\bigcup_{(e, k) \in \chi_{E}}\{(o, i) \mid o \in e(D), i \in o, i \in \mathcal{M}(k)\}
$$

- if the output schema of $E$ is $\{$ id $\}$, then $E(\operatorname{rep}(D))$ equals

$$
\bigcup_{(e, k) \in \chi_{E}}\left\{o \in e(D) \mid o \models \mathcal{M}^{*} k\right\} .
$$

Of course the global SA expression $E$ expressing the search query $q$ has output schema $\Sigma$ with $\{$ id $\} \subseteq \Sigma \subseteq\{$ id, item $\}$, and thus we can define $q$ in ASL as $\left(e_{1}\right.$ and $\left.k_{1}\right)$ or ...or $\left(e_{n}\right.$ and $\left.k_{n}\right)$ where $\chi_{E}=\left\{\left(e_{1}, k_{1}\right), \ldots,\left(e_{n}, k_{n}\right)\right\}$.

We construct $\chi_{E}$ by induction as follows:

$$
\begin{aligned}
& \chi_{T}:=\{(\star, \star)\} \\
& \chi_{\sigma_{l}(E)}:=\left\{(e, k \wedge l) \mid(e, k) \in \chi_{E}\right\} \\
& \chi_{\pi_{\mathrm{id}}(E)}:=\left\{(e \text { and } k, \star) \mid(e, k) \in \chi_{E}\right\} \\
& \chi_{E_{1} \cup E_{2}}:=\chi_{E_{1}} \cup \chi_{E_{2}} \\
& \chi_{E_{1}-E_{2}}:=\left\{\left(e_{1} \text { except } e_{2}, k_{1}\right),\left(e_{1}, k_{1} \wedge \neg k_{2}\right) \mid\right. \\
&\left.\left(e_{1}, k_{1}\right) \in \chi_{E_{1}},\left(e_{2}, k_{2}\right) \in \chi_{E_{2}}\right\} \\
& \chi_{\pi_{\text {id }}\left(E_{1} \ltimes \sim \pi_{\text {item }} E_{2}\right)}:=\left\{\left(e_{1} \text { and link }\left\langle k_{1} \sim k_{2}\right\rangle e_{2}, \star\right) \mid\right. \\
&\left.\quad\left(e_{1}, k_{1}\right) \in \chi_{E_{1}},\left(e_{2}, k_{2}\right) \in \chi_{E_{2}}\right\} \\
& \chi_{E_{1} \ltimes E_{2}}:=\left\{\left(e_{1} \text { and } e_{2} \text { and } k_{2}, k_{1}\right) \mid\right. \\
&\left.\left(e_{1}, k_{1}\right) \in \chi_{E_{1}},\left(e_{2}, k_{2}\right) \in \chi_{E_{2}}\right\}
\end{aligned}
$$

We conclude this section with a remark concerning conjunctions in join conditions. We have defined simlinks as link conditions involving just a single condition $k \sim l$. But link conditions involving a conjunction of two or more such conditions are also very natural. For example, the query link $\left\langle\left(k_{1} \sim_{1} l_{1}\right) \wedge\left(k_{2} \sim_{2} l_{2}\right)\right\rangle(e)$, applied to a dataspace $D$, would return those objects $o \in D$ for which there exists an object $o^{\prime}$ in $e(D)$ such that there exists a $k_{1}$-item $i \in o$ and an $l_{1}$-item $j \in o^{\prime}$ such that $i \sim_{1} j$, and there also exists a $k_{2}$-item $i \in o$ (not necessarily the same $i$ ) and an $l_{2}$-item $j \in o^{\prime}$ (not necessarily the same $j$ ) such that $i \sim_{2} j$. We can show (proof omitted) that such conjunctive join conditions bring us outside the semijoin algebra. Nevertheless, it is still possible to define a fragment of the relational algebra and prove a characterization along the lines of Theorem 21. We omit the details.

\section{ATTRIBUTE-VALUE DATASPACES}

Let us now apply our abstract theory to the concrete setting of dataspaces as they have been investigated in the literature $[11,12,17]$. In this setting, items are attribute-value pairs as in the relational setting of Section 3, so $\mathcal{I}=\Sigma \times \mathcal{V}$. The important difference, however, is that the universe of attributes $\Sigma$ can now be infinite; there is no longer a fixed finite relation schema. Of course each dataspace is finite, so in each dataspace just a finite number of attributes will occur, but these attributes can vary from dataspace to dataspace and even from object to object. Moreover, attributes can appear multiple times in the same object, with different values. So, an object really is just a nonempty finite set of items, without any restriction.

The dataspace models in the literature $[11,12,17]$ give each object an id, and naturally represent a dataspace $D$ as a set of triples $\{(o, a, v) \mid o \in D$ and $(a, v) \in o\}$. We argue that our view of a dataspace as just a set of objects is equivalent. Indeed, we just showed how to go from our representation to the set of triples; if one wants to go in the converse direction, the only difficulty one may encounter is that there might be two object id's in the triple set with exactly the same set of associated $(a, v)$ pairs. In that case we can add an explicit id attribute to the objects, so that the sets become distinct. Having explicit id attributes is also necessary when we need to represent links between objects based on ids. We will see an example of such linking later (Example 27).

The next question is what would be an interesting universe $\mathcal{K}$ of keywords. Surely we already want all literal keywords $(a, v)$ to be present (as in the classical relational case), so that we can formulate basic queries like 'retrieve all persons who live in Belgium, like the beer Duvel, but do not like the beer Heineken': ${ }^{2}$

$$
\begin{array}{r}
((\text { country: Belgium }) \text { and (likes: Duvel })) \\
\text { except (likes: Heineken })
\end{array}
$$

We also want negation separately on attributes and values: for example, likes: $\neg$ Heineken retrieves objects containing a value for attribute likes that is different from Heineken, and $\neg$ likes: Heineken retrieves objects containing Heineken as the value of an attribute different from likes. Note that, since the set of attributes is not fixed, we cannot express the last example by a disjunction using all possible attributes other than likes. Similarly, we need wildcards on values as well as on attributes, so ( $\star$ : Belgium) retrieves all objects with value Belgium for some attribute. Finally, we need disjunctions such as (likes: $\neg($ Heineken $\vee$ Budweiser)). (We only need negated disjunctions; positive disjunctions can already be expressed in BSL using or.)

To sum up, we propose the following system of keywords for attribute-value $(\mathrm{AV})$ pairs.

Definition 22. An $A V$ keyword is a pair of one of the following forms:

$$
(a: v)|(a: \neg V)|(\neg A: v) \mid(\neg A: \neg V),
$$

where $a \in \Sigma, v \in \mathcal{V}$, and $A \subseteq \Sigma, V \subseteq \mathcal{V}$ are finite. AV keywords are interpreted as follows:

$$
\begin{aligned}
\mathcal{M}(a: v) & :=\{(a, v)\} \\
\mathcal{M}(a: \neg V) & :=\{(a, v) \mid v \in \mathcal{V}-V\} \\
\mathcal{M}(\neg A: v) & :=\{(a, v) \mid a \in \Sigma-A\} \\
\mathcal{M}(\neg A: \neg V) & :=\{(a, v) \mid a \in \Sigma-A, v \in \mathcal{V}-V\} .
\end{aligned}
$$

Note that $\neg \emptyset$ plays the role of a wildcard $\star$ on attributes or values, and then $(\star: \star)$ plays the role of the wildcard on pairs.

A first indication of the flexibility of this keyword system is that we do not need to add boolean combinations:

Proposition 23. BSL over $A V$ keywords is equivalent to $B S L$ over the boolean closure of $A V$ keywords.

\footnotetext{
${ }^{2}$ To improve readability, we will write attribute-value pairs $(a, v)$ as $(a: v)$, conforming more to a programming language-like syntax.
} 
Proof. It is readily verified that every boolean combination of AV keywords amounts to a disjunction of AV keywords. Such a disjunction can be expressed in BSL using or.

Since we have the wildcard, Corollary 6 applies, so we know that in the AV setting, BSL defines exactly all search queries that are $K$-distinguishing for some finite set $K$ of AV keywords. Recall that a search query $q$ is $K$-distinguishing if it is invariant under the equivalence $\simeq_{K}$ on objects (Definition 4). In the AV setting, we can formulate a more intuitive alternative to this equivalence relation, directly in terms of attributes and values, rather than AV keywords. The idea, similar to full genericity, is that only a finite set of attributes and values can be distinguished.

Definition 24. Let $W$ be a finite set of attributes and values. Let $\diamond$ be a "blank value", that is an arbitrary element not in $W$. For an attribute or value $x$, define

$$
\operatorname{blank}_{W}(x):= \begin{cases}x & \text { if } x \in W \\ \diamond & \text { otherwise. }\end{cases}
$$

We extend blank ${ }_{W}$ to AV pairs, objects, and dataspaces in the canonical, pointwise manner:

$$
\begin{aligned}
\operatorname{blank}_{W}(a, v) & \left.:=\operatorname{blank}_{W}(a), \operatorname{blank}_{w}(v)\right), \\
\operatorname{blank}_{W}(o) & :=\left\{\operatorname{blank}_{W}(a, v) \mid(a, v) \in o\right\}, \\
\operatorname{blank}_{W}(D) & :=\left\{\operatorname{blank}_{W}(o) \mid o \in D\right\} .
\end{aligned}
$$

Two objects $o_{1}$ and $o_{2}$ are called $W$-equivalent if $\operatorname{blank}_{W}\left(o_{1}\right)$ $=$ blank $_{W}\left(o_{2}\right)$. We denote this by $o_{1} \simeq_{W} o_{2}$. We now say that a search query $q$ is $W$-distinguishing if for any two dataspaces $D_{1}$ and $D_{2}$ and objects $o_{1} \in D_{1}$ and $o_{2} \in D_{2}$ that are $W$-equivalent, we have $o_{1} \in q\left(D_{1}\right)$ iff $o_{2} \in q\left(D_{2}\right)$.

Proposition 25. A search query is $K$-distinguishing for a finite set of $A V$ keywords $K$ if, and only if, it is $W$ distinguishing for a finite set of attributes and values $W$.

Proof. The crux of the matter is that if two objects are $K$ equivalent, for some finite set of AV keywords $K$, then they are $W$-equivalent, where $W$ is the finite set of attributes and values explicitly mentioned in the keywords in $K$. Conversely, if two objects are $W$-equivalent, for some finite set of attributes and values $W$, then they are $K$-equivalent, where $K$ is the finite set of all AV keywords that can be constructed using the elements in $W$.

Let us now turn to associative search in the AV context. What are the simrels needed to join objects in an AV dataspace? Focusing on equijoins, there are three natural possibilities: two AV pairs can be compared on their values, on their attributes, or on both together. So, for the set $\mathcal{S}$ of simrels in the AV setting, we will use the set $\{$ eq, eq-attr, eq-val\} defined as follows:

Definition 26. An eqrel (short for equality relation) is one of the three following simrels on AV pairs:

$$
\begin{aligned}
(a, v) \text { eq }(b, w) & \Leftrightarrow a=b \text { and } v=w, \\
(a, v) \text { eq-attr }(b, w) & \Leftrightarrow a=b, \\
(a, v) \text { eq-val }(b, w) & \Leftrightarrow v=w .
\end{aligned}
$$

Example 27. For example, the following is an expression in the associative search language ASL over AV keywords and eqrel simlinks:

$\operatorname{link}\langle($ name: $\star)$ eq-val (author: $\star)\rangle$

(published in : ICDT 2009)

It defines the query that retrieves all authors of objects published in ICDT 2009. More precisely, it retrieves all objects with a value for attribute name that equals a value for attribute author in an object containing the item (published in, ICDT 2009).

In other dataspace models based on attribute-value pairs $[11,12]$, objects are not joined using eqrel simlinks, but through explicit named links (edges) between objects. So there, a dataspace is not merely a set of objects, but a graph of objects. Using eqrel simlinks as we do, an explicit graph model is redundant. Indeed, as just illustrated in Example 27, named edges (for instance linking papers to their authors) can easily be represented using id attributes for objects and "pointer" attributes (name and author) having these ids as values.

We next show that the abstract bisimulation Lemma 13 can well be applied in the present AV setting as an aid to understand the limits of expressive power of ASL. For example, consider the generic equality selection query $q_{a=b}$, for two attributes $a$ and $b$, defined as follows:

$$
q_{a=b}(D)=\{o \in D \mid \exists v:(a, v) \in o \text { and }(b, v) \in o\}
$$

It is not immediately clear whether or not this query is definable in ASL in the AV setting; it turns out it is not:

Proposition 28. $q_{a=b}$ is not definable in ASL over eqrel simlinks and $A V$ keywords.

In a nutshell, the proof of Proposition 28 consist of showing that $q_{a=b}$ is not bisimulation invariant relative to any finite set of AV keywords and eqrel simlinks, and then invoking Lemma 13.

We conclude this section by returning to the equivalence between ASL and the semijoin algebra. Recall that in Section 5, we have defined the semijoin algebra to work on abstract dataspaces represented as binary relations over the schema $\{$ id, item $\}$. While the equivalence of ASL and SA ${ }^{\text {search }}$ (Theorem 21) can be directly applied to the AV setting, it is not so natural to store AV pairs in a single item column. It is more natural to represent AV dataspaces as sets of triples, i.e., as ternary relations over the schema $\{\mathrm{id}$, attr, val $\}$. Also the RDF query language SPARQL works over such ternary relations $[2,16,22]$; RDF graphs can also be viewed as a dataspace model [12].

So, it is worthwhile to define an alternative to $\mathrm{SA}^{\text {search }}$ working on ternary relations. An added simplification is that, since we are working with eqrel simlinks rather than simlinks based on general abstract simrels, we will no longer need the $\sim$-semijoin operator and will have enough with the standard natural semijoin.

Definition 29. The fragment $\mathrm{SA}^{\mathrm{AV}}$ of the semijoin algebra, defined on relations $T:\{$ id, attr, val $\}$, is defined by the following grammar:

$$
\begin{aligned}
E::= & T\left|\sigma_{\text {attr }=c}(E)\right| \sigma_{\text {val }=c}(E)|E \cup E| E-E \mid \\
& \pi_{\{\text {id }\}}(E) \mid \pi_{\{\text {id }\}}\left(E \ltimes \pi_{\alpha}(E)\right)
\end{aligned}
$$


where $c$ ranges over attribute and value constants, and $\alpha$ is either $\{$ attr $\},\{$ val $\}$, or $\{$ attr, val $\}$.

The semantics of $\ltimes$ is the standard natural semijoin on equality of common attributes.

We have the following analog of Theorem 21:

Theorem 30. A search query is definable in ASL over AV keywords and eqrel simlinks, if and only if it is definable in $\mathrm{SA}^{\mathrm{AV}}$, where we regard a dataspace $D$ as a ternary relation $\{(o, a, v) \mid o \in D$ and $(a, v) \in o\}$.

Proof. The only-if direction is similar to the proof of Theorem 21. AV keywords are expressed using combinations of constant selections using union and difference. Simlinks based on eq-attr, eq-val, or eq are expressed using semijoin with projection on the right (the set $\alpha$ in the syntax definition) equal to $\{$ attr $\},\{$ val $\}$, or $\{$ attr, val $\}$, respectively.

The if-direction is also similar, but we have the added complication that boolean combinations of keywords are not directly available in the AV setting. Yet, because of Proposition 23, we can simulate them in the language. Proposition 23 is only formulated for BSL, but the same argument holds for ASL, because link distributes over a disjunction of keywords used in a simlink: $\operatorname{link}\left\langle\left(\varphi_{1} \vee \varphi_{2}\right) \sim \psi\right\rangle(e)=$ link $\left\langle\varphi_{1} \sim \psi\right\rangle(e)$ or link $\left\langle\varphi_{2} \sim \psi\right\rangle(e)$. Semijoins are translated to simlinks using an eqrel depending on $\alpha$ : if $\alpha=\{$ attr $\}$ we use eq-attr, if $\alpha=\{$ val $\}$ we use eq-val, and if $\alpha=\{$ attr, val $\}$ we use eq.

\section{DISCUSSION}

Our goal has been to provide the beginnings of a theoretical foundation for search and associative search queries, motivated by the ubiquity of such queries in everyday information systems. Our approach has been to investigate search queries as restricted kinds of database queries, and to use the tools and the concepts already developed in the theory of database queries. (One of us has studied unrestricted, i.e., relationally complete querying of dataspacelike databases earlier [19].)

We have first presented a general abstract theory, then applied it to the concrete setting of attribute-value dataspaces. It would be interesting to conduct a similar application to the XML data model, for example, with XPath playing the role of relational algebra.

Mainly inspired by dataspaces [12], we have focused on selection queries, i.e., queries that always return a subset of the original objects. Current approaches to keyword search on structured databases [14, we give just one recent reference] return tuples of objects that are related by patterns. In the semijoin algebra one can express such patterns as long as they are not cyclic, but the patterns themselves cannot be returned. It remains to be investigated if and how our theory should be extended so that patterns can be returned. Of course, one can simply move to the full relational algebra, but then there is less news to discover.

It would also be interesting to look at a similar theory of search queries on RDF graphs, given their close similarity to AV dataspaces. It might be possible to specialize our semijoin algebra fragment $\mathrm{SA}^{\mathrm{AV}}$ into a fragment of SPARQL, which is some kind of specialized relational algebra for ternary relations (RDF triples) [13, 16, 22].
Acknowledgments The authors thank the ICDT 2009 reviewers for their constructive comments that helped improve the presentation of this article.

\section{REFERENCES}

[1] RDF primer. W3C Recommendation, February 2004.

[2] SPARQL query language for RDF. W3C Recommendation, January 2008.

[3] S. Abiteboul, R. Hull, and V. Vianu. Foundations of Databases. Addison-Wesley, 1995.

[4] A.V. Aho and J.D. Ullman. Universality of data retrieval languages. In Conference Record, 6th ACM Symposium on Principles of Programming Languages, pages 110-120, 1979.

[5] R. Baeza-Yates and B. Ribeiro-Neto. Modern Information Retrieval. Addison-Wesley, 1999.

[6] C. Beeri, T. Milo, and P. Ta-Shma. On genericity and parametricity. In Proceedings 15th ACM Symposium on Principles of Database Systems, pages 104-116, 1996.

[7] C. Beeri, T. Milo, and P. Ta-Shma. Towards a languages for the fully generic queries. In S. Cluet and R. Hull, editors, Database Programming Languages, Lecture Notes in Computer Science, pages 239-259. Springer, 1997.

[8] P. Blackburn, M. de Rijke, and Y. Venema. Modal Logic. Cambridge University Press, 2001.

[9] P. Blackburn, J. van Benthem, and F. Wolter, editors. Handbook of Modal Logic. Elsevier, 2007.

[10] A. Chandra and D. Harel. Computable queries for relational data bases. Journal of Computer and System Sciences, 21(2):156-178, 1980.

[11] J.-P. Dittrich and M.A. Vaz Salles. iDM: A unified and versatile data model for personal dataspace management. In Proceedings 32nd International Conference on Very Large Data Bases, pages 367-378, 2006.

[12] X. Dong and A. Halevy. Indexing dataspaces. In Proceedings ACM SIGMOD International Conference on Management of Data, pages 43-54, 2007.

[13] G.H.L. Fletcher. An algebra for basic graph patterns. Presented at the workshop on Logic in Databases, Rome, Italy, May 2008.

[14] K. Golenberg, B. Kimelfeld, and Y. Sagiv. Keyword proximity search in complex data graphs. In Proceedings ACM SIGMOD International Conference on Management of Data, pages 927-940, 2008.

[15] V. Goranko and M. Otto. Model theory of modal logic. In Blackburn et al. [9], chapter 5 .

[16] C. Gutierrez, C. Hurtado, and A. Mendelzon. Foundations of semantic web databases. In Proceedings 23rd ACM Symposium on Principles of Database Systems, pages 95-106, 2004.

[17] A. Halevy, M. Franklin, and D. Maier. Principles of dataspace systems. In Proceedings 25th ACM Symposium on Principles of Database Systems, pages $1-9,2006$.

[18] H.V. Jagadish et al. Making database systems usable. In Proceedings ACM SIGMOD International Conference on Management of Data, pages 13-24, 2007. 
[19] M. Jain, A. Mendhekar, and D. Van Gucht. A uniform data model for relational data and meta-data query processing. In Advances in Data Management '95, pages 146-165. Tata McGraw-Hill, 1995.

[20] D. Leinders, M. Marx, J. Tyszkiewicz, and J. Van den Bussche. The semijoin algebra and the guarded fragment. Journal of Logic, Language and Information, 14:331-343, 2005.

[21] D. Leinders and J. Van den Bussche. On the complexity of division and set joins in the relational algebra. Journal of Computer and System Sciences, 73(4):538-549, 2007.

[22] J. Pérez, M. Arenas, and C. Gutierrez. Semantics and complexity of SPARQL. In The Semantic Web: Proceedings $I S W C$, volume 4273 of Lecture Notes in Computer Science, pages 30-43. Springer, 2006.

[23] K.A. Ross and A. Janevski. Querying faceted databases. In Proceedings 2nd International Workshop on Semantic Web and Databases, volume 3372 of Lecture Notes in Computer Science, pages 199-218. Springer, 2005.

[24] J.D. Ullman. Principles of Database and Knowledge-Base Systems, volume II. Computer Science Press, 1989. 